\title{
Counterion correlations and attraction between like-charged macromolecules
}

\author{
Alexandre Diehl, ${ }^{1}$ Humberto A. Carmona, ${ }^{2}$ and Yan Levin ${ }^{3}$ \\ ${ }^{1}$ Departamento de Física, Universidade Federal do Ceará, Caixa Postal 6030, CEP 60455-760, Fortaleza, Ceará, Brazil \\ ${ }^{2}$ Departamento de Física e Química, Universidade Estadual do Ceará, CEP 60740-000 Fortaleza, Ceará, Brazil \\ ${ }^{3}$ Instituto de Física, Universidade Federal do Rio Grande do Sul, Caixa Postal 15051, CEP 91501-970, Porto Alegre, Rio Grande do \\ Sul, Brazil
}

(Received 17 November 2000; published 25 June 2001)

\begin{abstract}
A simple model is presented for the appearance of attraction between two like-charged polyions inside a polyelectrolyte solution. The polyions are modeled as rigid cylinders in a continuum dielectric solvent. The strong electrostatic interaction between the polyions and the counterions results in counterion condensation. If the two polyions are sufficiently close to each other their layers of condensed counterions can become correlated resulting in attraction between the macromolecules. To explore the counterion induced attraction we calculate the correlation functions for the condensed counterions. It is found that the correlations are of very short range. For the parameters specific to the double stranded DNA, the correlations and the attraction appear only when the surface-to-surface separation is less than $7 \AA$.
\end{abstract}

DOI: 10.1103/PhysRevE.64.011804

PACS number(s): 61.20.Qg, 87.15.Aa

\section{INTRODUCTION}

In the last few years a new phenomenon has attracted attention of the community of soft condensed matter physicists - the appearance of attraction between likecharged macromolecules in solutions containing multivalent ions. The problem is particularly fascinating because it contradicts our well established intuition that like-charged entities should repel [1-3]. The fundamental point, however, is that the electrolyte solutions are intrinsically complex systems for which many body interactions play a fundamental role.

The attraction between like-charged macromolecules is important for many biological systems. One particularly striking example is provided by the condensation of DNA by multivalent ions such as $\mathrm{Mn}^{2+}, \mathrm{Cd}^{2+}$, and various polyamines [4-6]. This condensation provides an answer to the long standing puzzle of how a highly charged macromolecule, such as the DNA, can be confined to a small volume of viral head or nuclear zone in procaryotic cell. Evidently, the multivalent ions serve as a glue that keeps the otherwise repelling like-charged monomers in close proximity [7]. In eukaryotic cells, the cytosol is traversed by a network of microtubules and microfilaments-rigid chains of highly charged protein ( $F$-actin) - which in spite of large negative charge agglomerate to form filaments of cytoskeleton [8]. The actin fibers are also an important part of the muscle tissue, providing a rail track for the motion of molecular motor myosin.

Although the nature of attraction between like-charged macromolecules is still not fully understood, it seems clear that the attractive force is mediated by the multivalent counterions [9-15]. A strong electrostatic attraction between the polyions and the oppositely charged multivalent counterions produces a sheath of counterions around each macromolecule. The condensed counterions can become highly correlated resulting in an overall attraction. It is important to note that the complex formed by a polyion and its associated counterions does not need to be neutral for the attraction to arise. Under some conditions the correlation induced attraction can overcome the monopolar repulsion coming from the net charge of the complexes.

Recently, a simple model was presented to account for the attraction between two lines of charges [16-18]. Each line had $Z$ discrete uniformly spaced monomers of charge $-q$, and $n \leqslant Z / \alpha$ condensed counterions of charge $\alpha q$, free to move along the rod. The net charge of such a polyioncounterion complex is $Q_{\text {eff }}=-(Z-\alpha n) q \leqslant 0$. Nevertheless, it was found that if $n>Z / 2 \alpha$ and $\alpha \geqslant 2$, at sufficiently short distances, the two like-charged rods would attract [17]. It was argued that the attraction resulted from the correlations between the condensed counterions and reached maximum at zero temperature. If $n<Z / 2 \alpha$ the force was always found to be repulsive.

Clearly, a one dimensional line of charge is a dramatic oversimplification of the physical reality. If we are interested in studying the correlation induced forces between real macromolecules their finite radius must be taken into account $[19,20,22]$. Thus, a much more realistic model of a polyion is a cylinder with a uniformly charged backbone $[19,20]$ or with an intrinsic charge pattern $[21,22]$ as, e.g., the helix structure of DNA molecule. Furthermore, the condensed counterions do not move along the line, but on the surface of the cylinder. Unfortunately, these extended models are much harder to study analytically.

In this paper we explore the effects of finite polyion diameter on the electrostatic interactions between the two polyions using Monte Carlo simulations. We find that the finite diameter and the associated angular degrees of freedom of condensed counterions significantly modify the nature of attraction. Thus, although there is still a minimum charge that must be neutralized by the counterions in order for the attraction to appear, this fraction is no longer equal to 50\% as was the case for the line of charge model. We find that the critical fraction depends on the valence of counterions and is less than $50 \%$ for $\alpha \geqslant 2$. For monovalent counterions no attraction is found. The crystalline structure of the condensed counterions, as first suggested by simulations of Gronbech- 


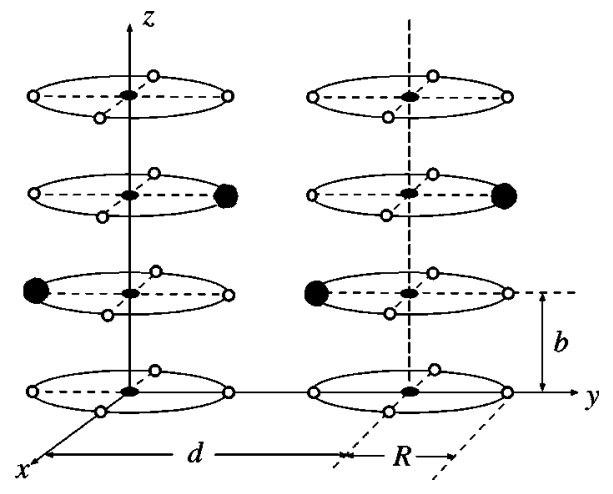

FIG. 1. Schematic depiction of the model: two polyions of $Z$ negative charges (small solid circles at the centers of rings), with radius $R$ and $n$ condensed counterions (large solid circles) each, are separated by distance $d$. The counterions are free to move along the $Z m$ positions (open circles) fixed on the rings located around each of the $Z$ monomers. In this sketch, $Z=4, n=2$, and $m=4$.

Jensen et al. [19] and Refs. [12,16,18,20], is also not very obvious. In particular we find very similar distributions of condensed counterions in the regime of attractive and repulsive interactions.

The structure of this paper is as follows. The model and the method of calculation are described in Sec. II. In Sec. III, we present the results of the simulations. The conclusions are summarized in Sec. IV.

\section{MODEL AND METHOD}

\section{A. The model}

The DNA model considered here is an extension of the one proposed earlier by Arenzon, Stilck, and Levin $[16,17]$. A similar model has been recently discussed by Solis and Olvera de la Cruz [20]. The polyions are treated as parallel rigid cylinders of radius $R$ and $Z$ ionized groups, each of charge $-q$, uniformly spaced-with separation $b$-along the principle axis, Fig. 1. Besides the fixed monomers, each polyion has $n \leqslant Z / \alpha$ condensed counterions with valence $\alpha$ and charge $\alpha q$, which are constrained to move on the surface of the cylinder. To locate a condensed counterion it is necessary to provide its longitudinal position, $z(0 \leqslant z<Z)$, and the transversal angle, $\theta(0 \leqslant \theta \leqslant 2 \pi)$. To simplify the calculations, the angular and the longitudinal degrees of freedom are discretized, see Fig. 1. The surface of the cylinder is subdivided into $Z$ parallel rings with a charged monomer at the center of each ring. Each ring has $m$ sites available to the condensed counterions, see Figs. 1 and 2. The hardcore repulsion between the particles requires that a site is occupied by at most one condensed counterion. The two polyions are parallel, with the intermolecular space treated as a uniform medium of dielectric constant $\epsilon$.

We introduce occupation variables $n_{i}^{k}$ for the two polyions, so that $i=1,2, \ldots, Z(m+1)$ and $k=1,2$. Thus, $n_{i}^{k}=1$ if the $i$ th site of the $k$ th polyion is occupied by a particle of valence $\alpha_{i}=\{-1, \alpha\}$ (negative core charge or counterion of valence $\alpha$, respectively), otherwise $n_{i}^{k}=0$. Note that the core charge is always "occupied," while the counterions are free
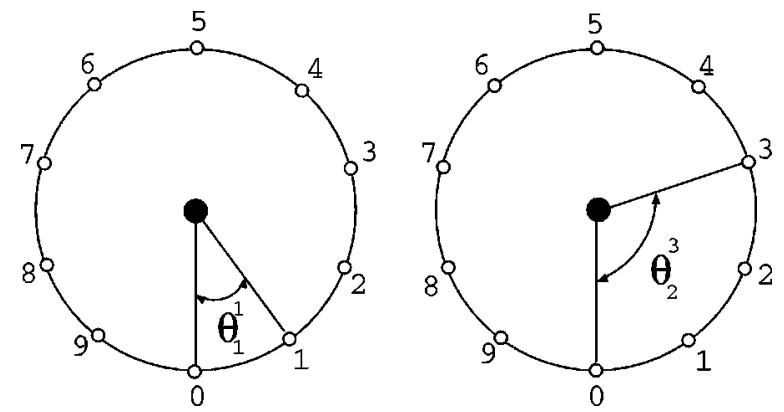

FIG. 2. The cross sectional view of the adjacent rings on the two polyions. The sites are labeled with integers $j=0,1, \ldots, m-1$ in the counter-clockwise direction. The angle between two consecutive sites is $\Delta \theta=2 \pi / m$. Here we show the angles $\theta_{1}^{1}$ of site $i=1$ on the polyion 1 , and $\theta_{3}^{2}$ of site $i=3$ on the polyion 2 .

to move between the $\mathrm{Zm}$ ring sites of each polyion. The Hamiltonian for the interaction between the two polyions is,

$$
\beta H=\xi \sum_{k, l} \sum_{i, j} \frac{\alpha_{i}^{k} n_{i}^{k} \alpha_{j}^{l} n_{j}^{l}}{\left|\boldsymbol{r}_{i}^{k}-\boldsymbol{r}_{j}^{l}\right|},
$$

with the $i \neq j$. All lengths are measured in units of $b$ (for DNA, $b=1.7 \AA$ ). The dimensionless quantity, $\xi=\beta q^{2} / b \epsilon$, is the Manning parameter, which for DNA is $\xi=4.17$. The partition function is obtained by tracing over all the possible values of $n_{i}^{k}$ consistent with the constraint of fixed number of counterions per polyion,

$$
\mathcal{Q}=\sum_{\left\{n_{i}^{k}\right\}} \exp (-\beta H)
$$

Clearly, this is a very crude model of the interaction between two macromolecules in a polyelectrolyte solution. The molecular nature of the solvent is ignored. Also the number of condensed counterions is fixed instead of being dependent on the separation between the particles. Nevertheless, we believe that this simple model can provide some useful insights for the mechanism of attraction in real polyelectrolyte solutions.

\section{B. The observables}

We are interested in statistical averages of observables such as the energy and the force between the two polyions. Furthermore, to understand the nature of the interaction between the two macromolecules it is essential to study the correlations between the condensed counterions on the two polyions.

The force is obtained from the partition function in Eq. (2), $b \beta \boldsymbol{F}=-\boldsymbol{\nabla}(\ln \mathcal{Q})$. From symmetry, only the $y$ component is different from zero.

For finite macromolecules the symmetry between the two polyions cannot be broken [18]. Hence it is impossible to produce a true crystalline order in a finite system at nonzero temperature. Since within our simplified model the two polyions have exactly the same number of condensed counterions, the average angular counterion distribution $\left\langle n_{i}^{k}\left(z, \theta_{i}^{k}\right)\right\rangle$ 


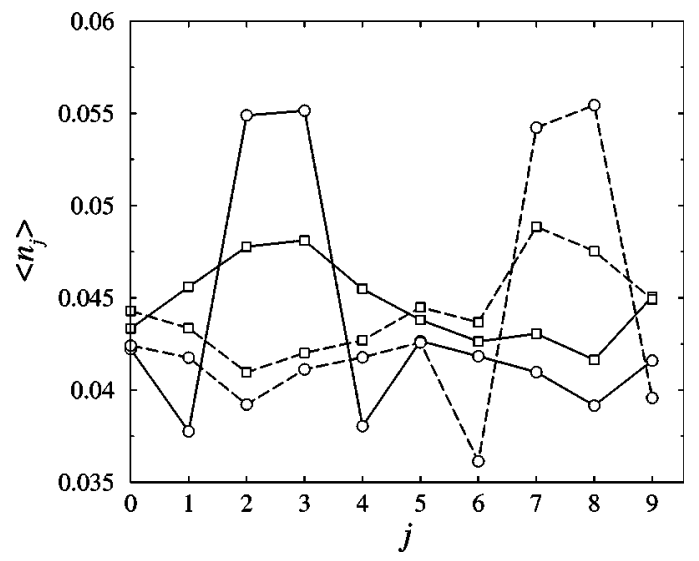

FIG. 3. Mean site occupation for the adjacent rings on the two polyions located at $z=9$. Each polyion has $Z=20$ and $n=7$. The distance between the centers of rings is $d / b=32.8$ (squares) and $d / b=16.65$ (circles). The solid line corresponds to the sites on the first polyion and the dashed lines are for the second polyion. The site label $j$ is the same as in Fig. 2. Note the almost perfect symmetry between the two macromolecules.

must be symmetric with respect to the midplane $y=d / 2$, see Fig. 1. The angle $\theta_{i}^{k}$ labels the site $i$ on polyion $k$, see Fig. 2 . Thus, $n_{3}^{2}\left(z, \theta_{3}^{2}\right)$ denotes the occupation variable for the site 3 on the ring $z$ located on polyion 2, with an angle of $3(2 \pi / m)$. Indeed, Fig. 3 shows that the density profiles are completely symmetric (up to fluctuations). In spite of this symmetry it is possible for the counterions on the two polyions to become highly correlated. Clearly, the strength of these correlations will depend on the product $\xi \alpha^{2}$ and the separation between the two macromolecules. Considering Fig. 2, it is evident that if the site 2 on the first polyion is occupied, the likelihood of occupation of the site 8 on the second polyion will be reduced.

To explore the nature of electrostatic correlations, we define a counterion-hole correlation function between the adjacent rings on the two polyions,

$$
\begin{aligned}
c_{z i j}^{12}= & \left\langle n_{i}^{1}\left(z, \theta_{i}^{1}\right)\left[1-n_{j}^{2}\left(z, \theta_{j}^{2}\right)\right]\right\rangle \\
& -\left\langle n_{i}^{1}\left(z, \theta_{i}^{1}\right)\right\rangle\left\langle\left[1-n_{j}^{2}\left(z, \theta_{j}^{2}\right)\right]\right\rangle .
\end{aligned}
$$

Here $\langle\cdots\rangle$ denotes the ensemble average. This function should be nonzero when sites on the two polyions are correlated, that is if one is occupied by a condensed counterion there is an increased probability of the second being empty.

Another quantity of interest is the ring-ring charge correlation function along the $z$ axis of the polyion $k$. To measure this, we first define the net charge of the ring centered on $z$,

$$
Q^{k}(z)=\alpha q \sum_{i=1}^{m} n_{i}^{k}\left(z, \theta_{i}^{k}\right)-q
$$

The charge correlation function between the rings of polyion is then,

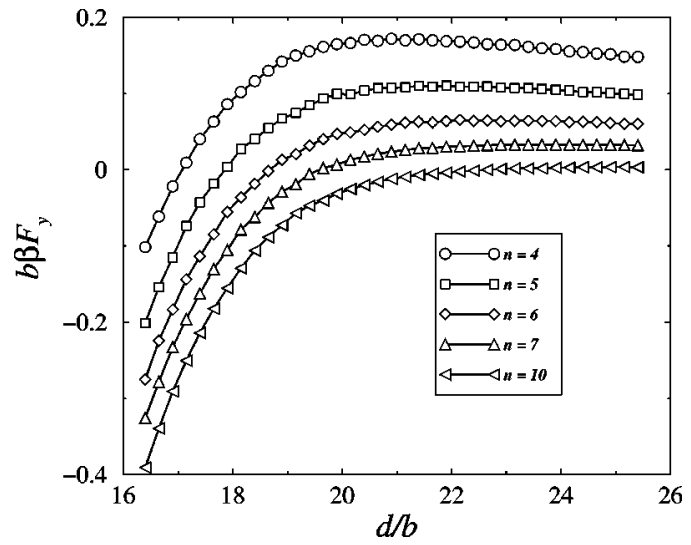

FIG. 4. Mean force in $y$ direction vs distance $d / b$ between the two DNA molecules $R / b=8.2, Z=20$, and $\xi=4.17$. The symbols indicate the number $n$ of divalent $(\alpha=2)$ condensed counterions.

$$
h^{Q Q}\left(z_{0}, z\right)=\left\langle Q^{k}\left(z_{0}\right) Q^{k}(z)\right\rangle-\left\langle Q^{k}\left(z_{0}\right)\right\rangle\left\langle Q^{k}(z)\right\rangle .
$$

We have chosen the reference point $z_{0}$ in the middle of the polyion in order to diminish the end effects.

\section{Simulations}

To calculate the force between the two polyions, we have performed a standard Monte Carlo simulation with the usual Metropolis algorithm [23]. First, one counterion on polyion 1 is randomly chosen and displaced to a vacant position on the same polyion. This move is accepted or rejected according to the standard detailed balance criterion [23]. We do not permit exchange of particles between the polyions. Next, the same is done for polyion 2. In one Monte Carlo step (MCS) all $2 n$ condensed counterions on the two polyions are permitted to attempt a move.

The long-ranged nature of the Coulomb interaction requires evaluation of all the pair interactions in Eq. (1) at every MCS. Due to the limited computational power available to us, we have confined our attention to relatively small systems with $Z<100$ and $m=10$. We have checked, however, that for $m=10$ the force has already reached the continuum limit and did not vary further with increase of $m$. Also we note that the "thermodynamic limit" is reached reasonably quickly, so that there is a good collapse of data already for $Z>50$, see Fig. 5. Two thousand MCS served to equilibrate the system, after which 500 samples were used to calculate the basic observables, namely, the mean force and the energy. To obtain the correlation functions, 5000 samples were used with 5000 MCS for equilibration.

\section{RESULTS AND DISCUSSION}

The simulations were performed for $\xi=4.17$ and $R / b$ $=8.2$, relevant for DNA. For monovalent counterions the simulation results indicate that the force is purely repulsive. This is in complete agreement with the experiments [1], which do not find any indication of DNA condensation for monovalent counterions.

For divalent counterions the force between the two complexes can become negative, indicating appearance of attrac- 


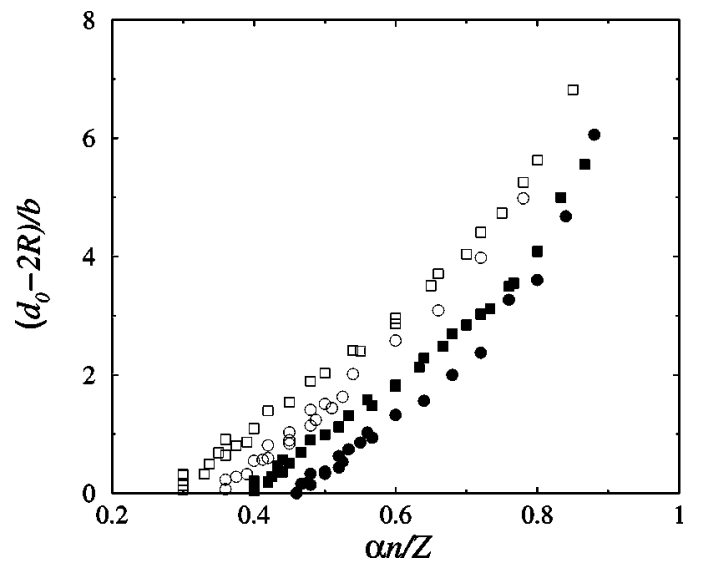

FIG. 5. The surface-to-surface separation below which the force becomes attractive, as a function of the number $n$ of condensed counterions, for valences $\alpha=2$ (full) and 3 (open), $\xi=2.283$ (circles) and 4.17 (squares), with $Z$ ranging from 50 to 100 .

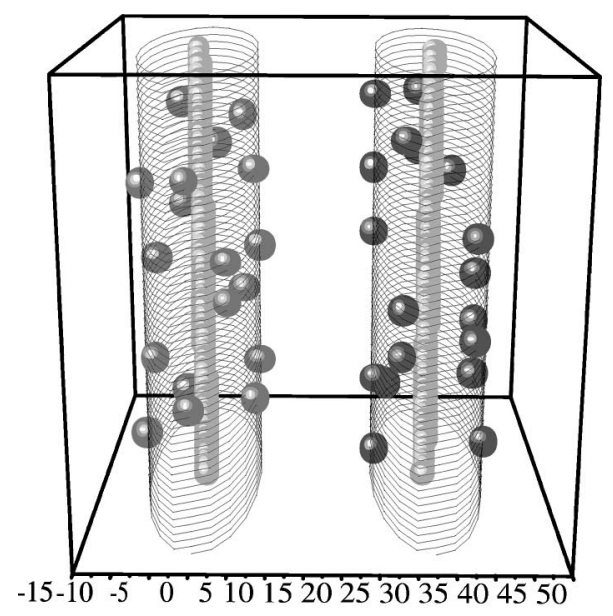

(a)

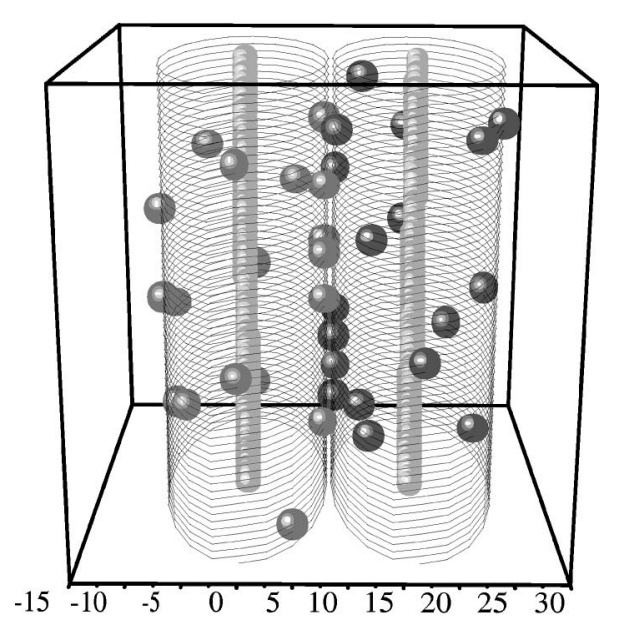

(b)

FIG. 6. Snapshots of two equilibrium configurations for (a) $d / b=32.8$ and (b) $d / b=16.8$, for two polyions with $Z=60$ and $n$ $=18$.

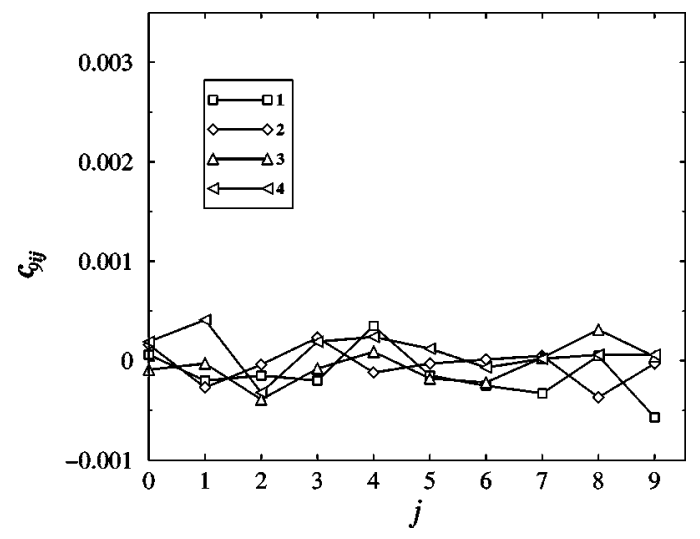

FIG. 7. Site-site correlation functions, Eq. (3), for the $i$ th site on the ring $z=9$ of the first polyion, with the $j$ th site on the $z=9$ ring of the second polyion. We consider only the correlations of the four "inner sites" of the first polyion $i=1,2,3,4$, (the four curves), with all the sites of the $z=9$ ring of the second polyion, $j=0,1, \ldots, m$ -1 , see Fig. 2. The total number of sites per ring is $m=10$. The parameters are as in Fig. 4 with $n=7$ and the separation between the two macromolecules is $d / b=32.8$. The graph shows that at this distance there are almost no correlations between the condensed counterions. From Fig. 4 we also see that the effective force is repulsive.

tion, Fig. 4. The range of attraction is larger than was found for the one dimensional line of charge model, Ref. [17].

Within the Manning theory [24] 88\% of the DNA's charge is neutralized by the divalent counterions. However, there are indications that even a larger fraction of DNA's charge can become neutralized by the multivalent ions if the counterion correlations are taken into account [14]. In this case the interaction is purely attractive, with the range of about $d / b \approx 20$ or $34 \AA$ ( $7 \AA$ surface-to-surface), Fig. 4 .

A minimum number of condensed counterions is necessary for attraction to appear. In Fig. 5 we present the surfaceto-surface separation, $\left(d_{0}-2 R\right)$, below which the force between the two complexes becomes negative (attractive), as a function of the number of multivalent counterions. For the case of DNA with divalent counterions $\alpha=2$, the attraction

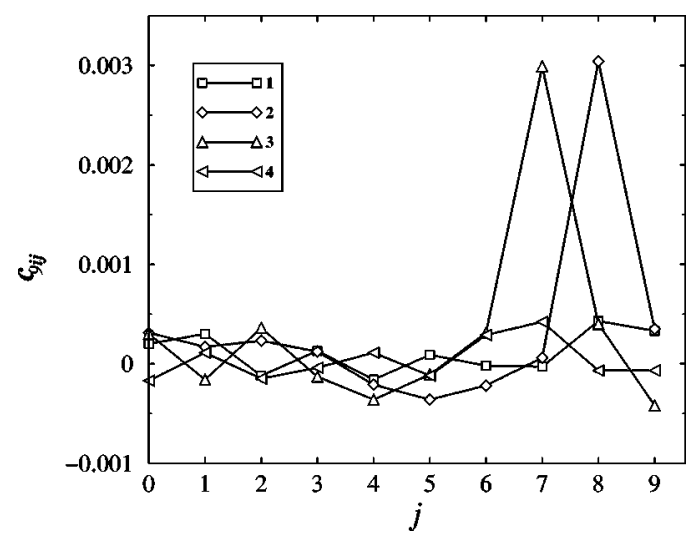

FIG. 8. Site-site correlation functions for separation $d / b$ $=16.65$, where, according to Fig. 4 , there is attraction. Note that sites 2 and 3 (open diamond and triangle, respectively) on polyion 1 are strongly correlated with sites 8 and 7 on polyion 2 , respectively. 


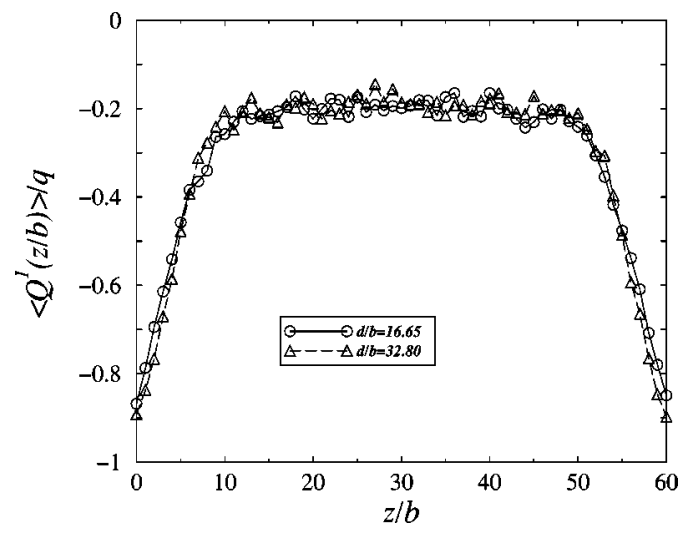

FIG. 9. The average charge of the ring centered on $z$ for two typical distances between the polyions. The parameters used in simulations are $Z=61, n=21, m=10, \alpha=2$, and $\xi=4.17$.

appears only if $40 \%$ of the core charge is neutralized. For $\alpha=3$ this fraction decreases to $30 \%$. Furthermore, decrease in the value of the Manning parameter, $\xi$, increases the minimum number of condensed counterions necessary for the attraction to appear. This is fully consistent with the fact that the attraction is mediated by the correlations between the condensed counterions. Since a rise in temperature tends to disorganize the system, the state of highest correlation between the condensed counterions corresponds to $T=0$ or $\xi$ $=\infty$.

The surface-to-surface distance at which the attraction first appears tends to zero as the number of condensed counterions is diminished. We find $d_{0}-2 R \sim\left[\mu-\mu_{c}(\alpha)\right]^{\nu}$, where the average counterion concentration is $\mu=n / Z$ and the critical fraction $\mu_{c}$ depends on the valence of condensed counterions $\alpha$. From Fig. 5 it is evident that $\nu=1$. This should be contrasted with the line of charge model Ref. [17], for which $\nu=1 / 3$.

In Fig. 6 we show two snapshots of the characteristic equilibrium configurations for (a) $d / b=32.8$ and (b) $d / b$ $=16.8$. Looking at these figures it is difficult to see something that would distinguish between them, both appear about the same. There is no obvious crystallization or transversal polarization suggested in previous studies $[19,20]$. Yet, the case (a) corresponds to the repulsive, while the case (b) corresponds to the attractive interaction between the polyions. To further explore this point, in Figs. 7 and 8 we present the site-site correlation function, Eq. (3), for macromolecules with $Z=20$ and $n=7$. For $d / b=32.8$ the surfaceto-surface distance between the two polyions is sufficiently large for their condensed counterions to be practically uncorrelated, Fig. 7. On the other hand, for $d / b=16.65$ strong correlations between the condensed counterions are evident, Fig. 8. Figure 8 shows that the sites two and three on the first polyion are strongly correlated with the sites seven and eight on the second polyion, respectively. It is these correlations between the adjacent sites on the two polyions that are responsible to the appearance of attraction between the two macromolecules when they are approximated, Fig. 4.

Finally, in Figs. 9 and 10 we show the average charge per ring and the charge correlation function along the polyion, in

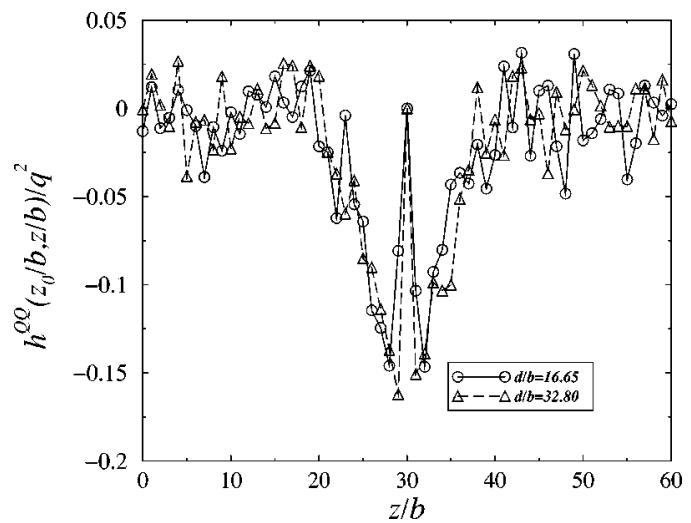

FIG. 10. Charge-charge correlation function between one site at the middle of polyion and the other $Z-1$ sites. The parameters are the same as in Fig. 9.

Eq. (5). Figure 9 demonstrates that the counterions are preferentially localized towards the center of the polyion. Furthermore, the distribution of charge along the polyion does not change much with the separation between the macromolecules. Thus, very similar charge profiles are found for $d / b$ $=32.8$, the distance at which force is repulsive, and $d / b$ $=16.8$, the distance at which force is attractive. Similar conclusion is reached for the charge-charge correlation function, Fig. 10. We see that there is a very strong anticorrelation between the net charge on the adjacent rings of the polyion that, however, rapidly decays with the separation between the rings.

\section{SUMMARY}

We have presented a simple model for polyion-polyion attraction inside a polyelectrolyte solution. It is clear from our calculations that the attraction results from the correlations between the condensed counterions. The thermal fluctuations tend to diminish the correlations, decreasing the amplitude of the attractive force. Consistent with the experimental evidence, the attraction exists only in the presence of multivalent counterions. Our simulations demonstrate that a critical number of condensed counterions is necessary for the appearance of attraction. The fraction of bare charge that must be neutralized for the attraction to arise depends on the valence of counterions. The larger the valence, the smaller the fraction of the bare polyion charger that must be neutralized for the attraction to appear. This result should be contrasted with the line of charge model [17] for which the critical fraction was found to be equal to $50 \%$, independent of the counterion charge.

\section{ACKNOWLEDGMENTS}

We thank J. J. Arenzon for helpful comments on simulations. This work was supported by $\mathrm{CNPq}$ (Conselho Nacional de Desenvolvimento Científico e Tecnológico) and FINEP (Financiadora de Estudos e Projetos), Brazil. 
[1] V.A. Bloomfield, Biopolymers 31, 1471 (1991).

[2] R. Podgornik, D. Rau, and V.A. Parsegian, Biophys. J. 66, 962 (1994).

[3] J. Israelachvili, Intermolecular and Surface Forces (Academic Press, London, 1985).

[4] D.C. Rau, and V.A. Parsegian, Biophys. J. 61, 260 (1992).

[5] J.G. Duguid, V.A. Bloomfield, J.M. Benevides, and G.J. Thomas, Jr., Biophys. J. 69, 2633 (1995).

[6] D.A. Knoll, M.D. Fried, and V.A. Bloomfield, in DNA and its Drug Complexes, edited by R.H. Sarma and M.H. Sarma (Adenin Press, New York, 1998).

[7] E. Raspaud, M. Olvera de la Cruz, J.-L. Sikorav, and F. Livolant, Biophys. J. 74, 381 (1998).

[8] J.X. Tang, S. Wong, P. Tran, and P. Janmey, Ber. Bunsenges. Phys. Chem. 100, 1 (1996).

[9] J. Ray and G.S. Manning, Langmuir 10, 2450 (1994).

[10] B.-Y. Ha, and A.J. Liu, Phys. Rev. Lett. 79, 1289 (1997).

[11] B.-Y. Ha, and A.J. Liu, in Physical Questions Posed by DNA Condensation, to appear in Physical Chemistry of Polyelectrolytes, edited by T. Radeva (Marcel Dekker, New York, 2000).

[12] I. Rouzina and V. Bloomfield, J. Chem. Phys. 100, 9977 (1996); B.I. Shklovskii, Phys. Rev. Lett. 82, 3268 (1999).
[13] B.I. Shklovskii, Phys. Rev. E 60, 5802 (1999).

[14] V.I. Perel and B.I. Shklovskii, Physica A 274, 5802 (1999).

[15] T.T. Nguyen, I. Rouzina, and B.I. Shklovskii, J. Chem. Phys. 112, 2562 (2000).

[16] Y. Levin, J.J. Arenzon, and J.F. Stilck, Phys. Rev. Lett. 83, 2680 (1999).

[17] J.J. Arenzon, J.F. Stilck, and Y. Levin, Eur. Phys. J. B 12, 79 (1999).

[18] J.J. Arenzon, Y. Levin, and J.F. Stilck, Physica A 283, 1 (2000).

[19] N. Gronbech-Jensen, R.J. Mashl, R.F. Bruisma, and M.W. Gelbart, Phys. Rev. Lett. 78, 2477 (1997).

[20] F.J. Solis and M. Olvera de la Cruz, Phys. Rev. E 60, 4496 (1999).

[21] A.A. Kornyshev and S. Leikin, Phys. Rev. Lett. 82, 4138 (1999).

[22] E. Allahyarov and H. Löwen, Phys. Rev. E 62, 5542 (2000).

[23] M.P. Allen, and D.J. Tildesley, Computer Simulations of Liquids (Clarendon, Oxford, 1987).

[24] G.S. Manning, J. Chem. Phys. 51, 924 (1969); Q. Rev. Biophys. 11, 179 (1978). 